\title{
MDCT in Characterizing Gall Bladder Carcinoma: Experience From an Endemic Region
}

\author{
Niharika Prasad ${ }^{1}$, Amit Goyal ${ }^{2}$ \\ ${ }^{1}$ Senior Resident, Radiodiagnosis, Institute of Liver and Biliary Sciences (ILBS), ${ }^{2}$ Medical Officer, SDMC, New Delhi, India \\ Corresponding author: Niharika Prasad, Veeraytan Netralaya, V.I.P Road, Ashok Nagar, Pokharia, Begusarai, Bihar (851101), \\ India
}

DOI: 10.21276/ijcmsr.2018.3.2.32

How to cite this article: Niharika Prasad, Amit Goyal. MDCT in characterizing gall bladder carcinoma: experience from an endemic region. International Journal of Contemporary Medicine Surgery and Radiology. 2018;3(2):B133-B136.

\section{A B S T R A C T}

Introduction: With the technical advancements in detection and treatment of gall bladder malignancy, it is essential that detailed pre-operative imaging should be available to guide the surgeon. Although its incidence is high in certain Indian states, the utility of triple-phase MDCT has infrequently been described in literature. The study aimed to determine the utility of multi-phase MDCT in the staging and resectability of gallbladder carcinoma.

Material and Methods: Fifty patients with suspected gall bladder carcinoma on routine sonography were subjected to multiphasic MDCT in AIIMS Patna within six months duration. All three planes were assessed for evaluation of the vascular invasion and anatomy. Tumour enhancement and washout characteristics, loco regional and distant metastasis were documented. Attempts to differentiate carcinoma from benign mimickers like polyps, cholecystitis and adenomyomatosis were made.

Results: The sensitivity and specificity of MDCT in detection of carcinoma of gall bladder was $92.8 \%$ and $96.7 \%$ respectively. It is superior to ultrasonography in detecting early stage carcinoma as well as vascular and bile duct infiltration.

Conclusion: Triple-phase MDCT with 3D reconstruction is a comprehensive imaging technique for staging gallbladder carcinoma and determining the vascular road map before surgery.

Keywords: Triple-phase, Sonography, 3D Reconstruction, Polyps, Adenomyomatosis, Cholecystitis, Roadmap

\section{INTRODUCTION}

Incidence of gall bladder carcinoma is highest in Asian countries and this had increased recently due to improved detection rates. Cholecystectomy is increasingly being performed by laparoscopic method and more cases are diagnosed by endoscopy and advanced imaging techniques. Gall bladder carcinoma is more common in females. Other risk factors are advanced age, cholelithiasis, porcelain gallbladder, gallbladder polyps, chronic infection, and smoking. Most common complaints are right upper quadrant/ epigastric pain or discomfort, nausea and weight loss. Few cases are also picked up incidentally on cholecystectomy for calculi. Approximately 90\% of Gall Bladder cancers have accompanying cholelithiasis. ${ }^{1}$

Adenocarcinoma is the most common histologic type, accounting for $98 \%$ of all gallbladder tumours with almost a third of cases presenting with distant metastasis. ${ }^{2}$ With the aid of MDCT, the surgeon gets a roadmap of arterial and venous anatomy and can determine plane of resection for segmentectomy. Another advantage of cross sectional imaging is to warn the operating surgeon of anatomical variations. ${ }^{3}$ The diagnosis is usually in advanced stage and imaging should aim to pick it up early to reduce patient morbidity and mortality. Computed tomography (CT)
Staging system most commonly used is American Joint Committee on Cancer (AJCC) TNM system.

The different types of presentations of gall bladder(GB) carcinoma on CT are- a) Mass Occupying or Replacing the Gallbladder Lumen, b) Focal or Diffuse Asymmetric Wall Thickening and c) Intraluminal Polyp. ${ }^{4}$ The one layered pattern with a heterogeneously enhancing thick layered pattern (Type 1) was significantly associated with gall bladder cancer ( $p$ value 0.001). The sensitivity and specificity of Type 1 enhancement pattern on CT for predicting the Gall Bladder (GB) malignancy were $90.476 \%$ and $97.43 \%$ respectively, by a study in $2017 .{ }^{5}$

The radiologic differential diagnosis includes the more common inflammatory conditions of the gallbladder, benign polyps, xanthogranulomatous cholecystitis, adenomyomatosis, other hepatobiliary malignancies, and metastasis. ${ }^{6}$

The study aimed to determine the utility of multi-phase MDCT in the staging and resectability of gallbladder carcinoma. It attempts to explore pattern of spread of neoplasm and how to recognise it at an early, treatable stage. A secondary objective was to retrospectively differentiate gall bladder neoplasm from other close, benign mimickers.

\section{MATERIAL AND METHODS}

A hospital based, cross-sectional study was done from 
August 2017 to January 2018 in the department of Radiodiagnosis, All India Institute of Medical Sciences, (AIIMS) Patna. Contrast enhanced computed tomography (CECT) images of 50 patients showing ultrasound findings suspicious of malignancy were included. Each patient's personal data, clinical information and histopathological findings were recorded.

All patients above the age of 18 years were included in study including both males and females. Informed consent was taken from all patients. Exclusion criteria included post cholecystectomy status, diffuse GB wall edema due to systemic disease, severely contracted gall bladder on CT or dense echogenic sludge completely filling lumen, uncooperative patients, patients with contrast allergy and those not willing to give consent.

Non contrast images were obtained from the lower thoracic level up to the pelvic region covering the entire abdomen. The NCCT scans enabled detection of calculi and wall calcifications, following which, contrast enhanced multiphase imaging was performed. A triple phase protocol was used with images acquired on a 256 slice dual source Siemens SOMATOM Flash CT scanner. Three phases- 1) Arterial (20-25 seconds), 2) Porto venous (60-70 seconds) and 3) Delayed phases (5-10 seconds) were performed. All patients were administered $1 \mathrm{~mL} / \mathrm{kg}$ of intravenous contrast material at an injection rate of $4-6 \mathrm{~mL} / \mathrm{sec}$. The phases were obtained using SmartPrep technique. Multiplanar (coronal, sagittal and oblique), MIP (maximum intensity projection) and MinIP (minimum intensity projection) were obtained
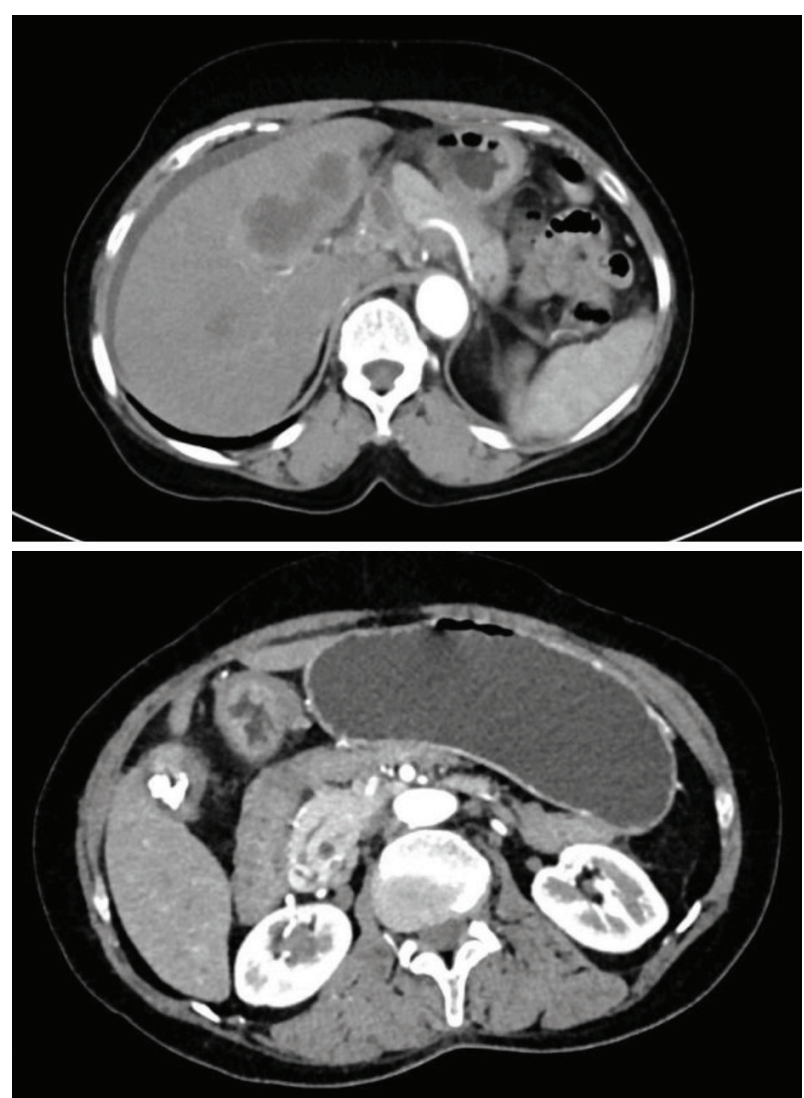

Figure-1: Types of gall bladder carcinoma on CECTnecrotic mass type (above), enhancing wall thickening with GB calculi (below) and analysed.

The location of the tumour, locoregional spread, and metastasis were noted and resectability of the tumour was determined. Degree of enhancement and washout was documented by drawing ROI within lesion in arterial, portal, venous and delayed phases. In particular, infiltration of portal vein or hepatic artery, involvement of the portal vein or hepatic artery branches; extensive infiltration of the colon, duodenum, or pancreas and the presence of peritoneal metastases was looked for and described to determine resectability. Loss of surrounding normal cuff of fat plane around vessels was analysed to check for infiltration. All biopsy proven cases of carcinoma of gall bladder during the study period were retrospectively analysed to calculate sensitivity and specificity of MDCT in detection of GB malignancy.

\section{RESULTS}

The patients diagnosed to be GB carcinoma on histology consisted of 22 women and 18 men out of which $88 \%$ were over 50 years of age. Majority ( $76 \%$ of total) were found to be stage III or IV. $60 \%$ of the early stage malignancy presented with isolated focal enhancing GB wall thickening, with or without calculi, latter being more common. In $24 \%$ of study subjects, CECT led to upstaging ultrasound findings due to improved detection of vessel wall infiltration (Portal vein,

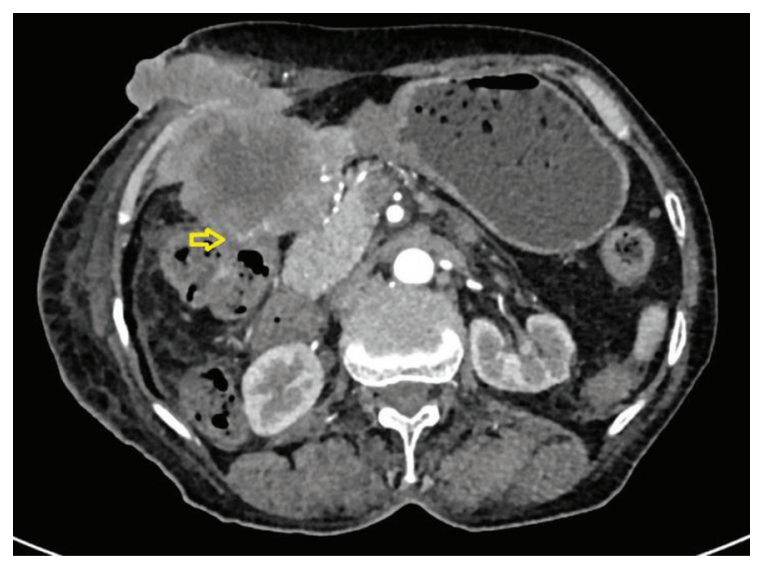

Figure-2: Advanced stage gall bladder mass lesion is seen to infiltrate abdominal wall, head of pancreas medially as well as adjacent wall of duodenum (arrow shows loss of fat planes with duodenum).

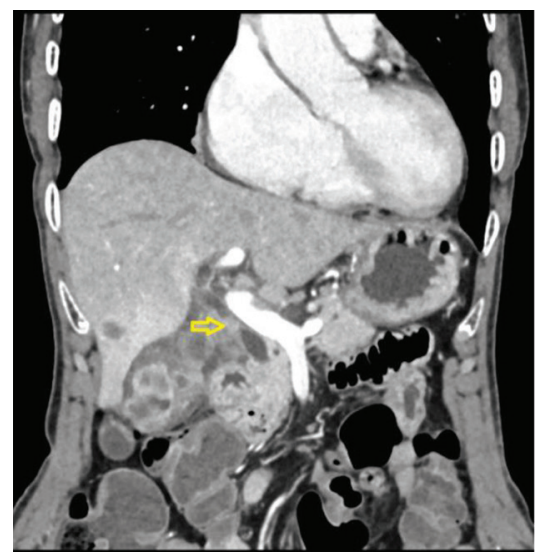

Figure-3: Portovenous phase- Infiltration of CBD (arrow) by gall bladder mass lesion, with invasion of porta 


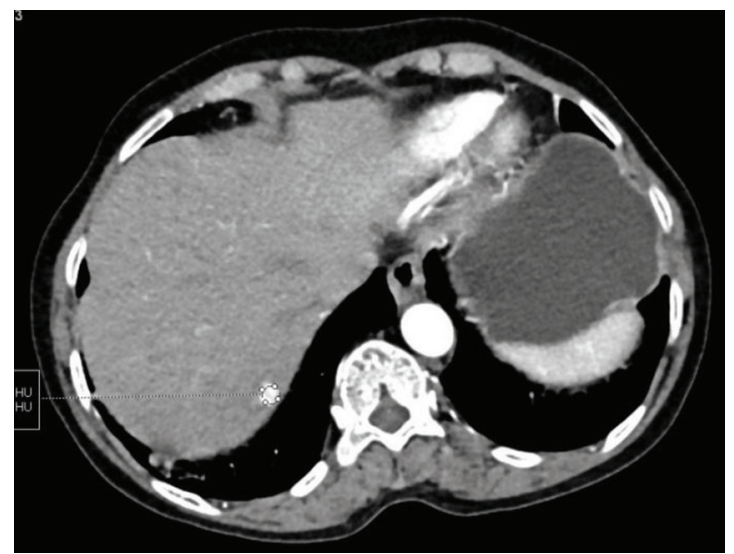

Figure-4: Hypervascular liver metastasis such as above case is typical of adenocarcinoma. Small metastasis may be missed on ultrasound examination.
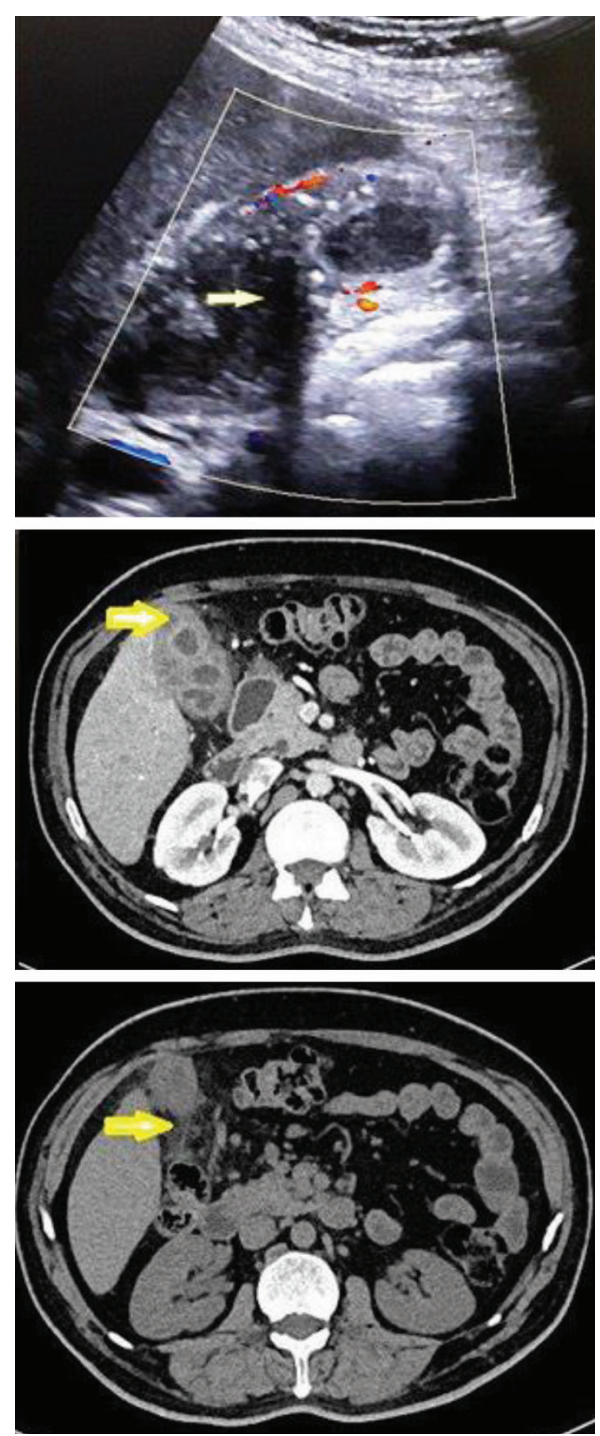

Figure-5: Xanthogranulomatous cholecystitis mimicking neoplasm: Young male with uncontrolled diabetes, mass like hypoechoic lesion which appears to infiltrate GB wall. CECT image (middle) shows hypo attenuating nodular, non-enhancing areas in GB wall. NECT image shows fat stranding - s/o inflammatory changes, extending to duodenum and colon. A small gall bladder calculus was also present (not shown).

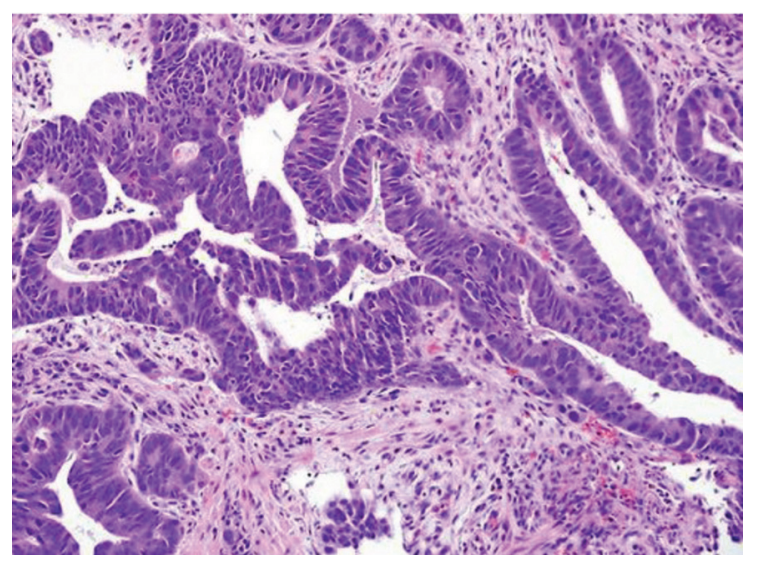

Figure-6: Histopathological image shows abundance of glandular elements in one of the cases of adenocarcinoma of gall bladder.

hepatic artery, hepatic vein and others) or common bile duct. Sub centimetre portal lymph nodes were better visualised on CT which were not seen on ultrasound in doubtful cases of chronic cholecystitis versus neoplasm. $48 \%$ of the adenocarcinoma cases showed hyper vascular liver metastasis. Nine out of the total study, presented with involvement of duodenum, colon, peritoneum or other systemic metastasis. Two of the cases were successfully distinguished from primary liver neoplasm/cholangiocarcinoma only on biopsy. The sensitivity and specificity of MDCT in detection of carcinoma of gall bladder were $92.8 \%$ and $96.7 \%$ respectively.

\section{DISCUSSION}

The most common biliary tract malignancy, Carcinoma of Gall Bladder is overall still rare. High incidence of GB carcinoma has been found in Northern India especially the gangetic belt. ${ }^{10}$

With increased rates of cholecystectomy which are attributable to advance techniques in laparoscopy, endoscopy, histopathology and imaging the incidence of GB carcinoma is on the rise.

There are multiple causative factors among which cholelithiasis is seen in 69 to $85 \%$ of patients in different regions, mucosal inflammation due to gallstones is believed to play a role in carcinogenesis. ${ }^{7}$ Adenocarcinomas account for $90 \%$ of gallbladder carcinomas and are characterized by glands lined by cuboidal or columnar cells, which may contain mucin. They may be well, moderately, or poorly differentiated. There are several recognized histologic variants of adenocarcinoma: papillary, intestinal, mucinous, signet-ring cell, and clear cell type. ${ }^{8}$

Besides GB adenocarcinoma, differential diagnosis of gallbladder wall thickening includes the post-prandial state, acute and chronic cholecystitis, cholesterol polyps and many other relatively uncommon conditions.

The first investigation is usually an abdominal ultrasound examination such as in this study which is also the preferred modality in follow up cases. As adenomatous or hyperplastic cholesterol polyps and sludge can resemble early stage neoplasm turning the patient and scanning was practised in such cases. At sonography, if movement of a polypoidal lesion occurs with a change of the patient's position, then 
a pseudotumor of biliary sludge or clot can be diagnosed. ${ }^{9}$ In simple polyps, the enhancement was found to be same as rest of the gall bladder wall on contrast-enhanced CT. The focal wall thickening adjacent polyp and implantation base thickening described in literature was not found to be a useful sign in differentiation from neoplasm. However, follow-up imaging to look for interval change in size proved beneficial.

In one young, diabetic patient in this study (case images) xanthogranulomatous cholecystitis is seen to appear like GB carcinoma. This was distinguished on CT by heterogeneous wall enhancement with continuous linear enhancement of the mucosa and hypo attenuating intramural nodules.

In another patient, adenomyomatosis was diagnosed on ultrasound by small hyperechogenic reverberating intramural foci -"comet tail" sign and further CECT showed small cystic areas or in the wall corresponding to dilated 'Rokitansky sinuses'.

Diffuse asymmetric wall thickening with enhancement in arterial phase and washout in subsequent portovenous phase (by 10-15 HU) was found to characteristic of GB adenocarcinoma in $>85 \%$ of patients in this study. In two of the cases it also revealed very small calculi which were missed on ultrasound. Early cases showed subcetimeter portal nodes while few late stage GB malignancies presented with biliary duct dilatation and infiltration of anterior surface of liver with or without metastasis. In one of the advanced cases, there was perforation of GB wall, forming fistula communicating with duodenum. Air foci seen within GB mass in such a case can resemble emphysematous cholecystitis. Coronal sections are more useful than axial ones in such cases to delineate extent and colon involvement.

Thus, CECT with reconstruction of images should be performed in patients suspected of GB carcinoma or detected so on ultrasound for better delineation of extent, regional involvement and metastasis. It also aids in staging, identifying anatomical variants and effective preoperative panning. MRI and MRCP (Magnetic Resonance Cholangiopancreatography) has a limited role and is required in very few, doubtful cases. Further, T1-hyperintensity can interfere with the diagnosis of gallbladder adenomyomatosis as it is the consequence of concentrated biliary content, cholesterol crystals or calcifications. Also, polyps have variable appearance on MR, which often overlap with $\mathrm{GB}$ malignancy. ${ }^{11}$

Similarly, PET (Positron emission tomography) is not reliably accurate in excluding early gallbladder neoplasms and may be unsatisfactory in early lesions measuring less than $1 \mathrm{~cm} .{ }^{12}$

\section{CONCLUSION}

Thus MDCT due to its wide availability and good accuracy remains gold standard in staging and preoperative evaluation of Gall Bladder Adenocarcinoma and has proved its utility in differentiating it from other pathologies.

\section{Acknowledgement}

I am grateful to Dr. Sanjay Purushothama, Assistant Professor, Department of Radio-Diagnosis, Mysore Medical College and Research Institute, Mysore and Dr. Subhash
Kumar, Associate Professor, AIIMS Patna, Bihar for their guidance and encouragement.

\section{REFERENCES}

1. Oddsdottir M, Hunter JG. Gall Bladder and the extrahepatic billiary sytem. In: Brunicardi FC, Andersen DK, Billiar TR, Dunn DL, Hunter JG, Pollock RE, editors. Schwartz's principle of surgery (8th ed.). New York: McGraw Hill; 2005.1187-1219. 2009; 9:63.

2. Rani Kanthan, Jenna-Lynn Senger, Shahid Ahmed, and Selliah Chandra Kanthan. Gallbladder Cancer in the 21st Century. Journal of Oncology, 2015.

3. Naveen Kalra, Sudha Suri, Rajesh Gupta, S. K. Natarajan, Niranjan Khandelwal, J. D. Wig, Kusum Joshi. MDCT in the Staging of Gallbladder Carcinoma. AJR:186, March 2006

4. Alessandro Furlan, James V. Ferris, Keyanoosh Hosseinzadeh, Amir A. Borhani. Gallbladder Carcinoma Update: Multimodality Imaging Evaluation, Staging, and Treatment Options. AJR:191, November 2008

5. Manoj Mathur et al., Imaging Evaluation of Enhancement Patterns of Flat Gall Bladder Wall Thickening and its Correlation. Journal of Clinical and Diagnostic Research. 2017;11(4):TC07-TC11.

6. Angela D. Levy, Linda A. Murakata, Charles A. Rohrmann, Jr. Gallbladder Carcinoma: RadiologicPathologic Correlation; Radio Graphics 2001; 21(1):295-314

7. Christine S. M. Lau, Aleksander Zywot, Krishnaraj Mahendraraj, Ronald S. Chamberlain. Gallbladder Carcinoma in the United States; Hindawi, HPB Surgery Volume 2017.

8. Albores-Saavedra J, Henson DE, Sobin LH. WHO histological typing of tumors of the gallbladder and extrahepatic bile ducts Berlin, Germany: SpringerVerlag, 1991.

9. Furlan A, Ferris JV, Hosseinzadeh K, Borhani AA. Gallbladder carcinoma update: multimodality imaging evaluation, staging, and treatment options. AJR Am J Roentgenol. 2008;191(5):1440-7.

10. Shukla VK, Khandelwal C, Roy SK,Vaidya MP. Primary carcinoma of the gallbladder: A review of a 16 year period at the university hospital. J Surg Oncol. 1985;28(3): 32-35

11. Timothy McKnight, D.O.,1 and Ankit Patel, D.O. Gallbladder Masses: Multimodality Approach to Differential Diagnosis; J Am Osteopath Coll Radiol 2012; Vol. 1, Issue 4

12. Bonatti M, Vezzali N, Lombardo F, et al. Gallbladder adenomyomatosis: imaging findings, tricks and pitfalls. Insights into Imaging. 2017;8(2):243-253.

\section{Source of Support: Nil; Conflict of Interest: None}

Submitted: 05-05-2018; Accepted: 04-06-2018; Published online: 15-06-2018 